\title{
Effects of case management in community aged care on client and carer outcomes: a systematic review of randomized trials and comparative observational studies
}

\author{
Emily Chuanmei You ${ }^{1 *}$, David Dunt ${ }^{1 *}$, Colleen Doyle ${ }^{2,3}$ and Arthur Hsueh ${ }^{1}$
}

\begin{abstract}
Background: Case management has been applied in community aged care to meet frail older people's holistic needs and promote cost-effectiveness. This systematic review aims to evaluate the effects of case management in community aged care on client and carer outcomes.

Methods: We searched Web of Science, Scopus, Medline, CINAHL (EBSCO) and PsycINFO (CSA) from inception to 2011 July. Inclusion criteria were: no restriction on date, English language, community-dwelling older people and/or carers, case management in community aged care, published in refereed journals, randomized control trials (RCTs) or comparative observational studies, examining client or carer outcomes. Quality of studies was assessed by using such indicators as quality control, randomization, comparability, follow-up rate, dropout, blinding assessors, and intention-to-treat analysis. Two reviewers independently screened potentially relevant studies, extracted information and assessed study quality. A narrative summary of findings were presented.

Results: Ten RCTs and five comparative observational studies were identified. One RCT was rated high quality. Client outcomes included mortality (7 studies), physical or cognitive functioning (6 studies), medical conditions (2 studies), behavioral problems (2 studies), unmet service needs (3 studies), psychological health or well-being (7 studies), and satisfaction with care (4 studies), while carer outcomes included stress or burden (6 studies), satisfaction with care (2 studies), psychological health or well-being (5 studies), and social consequences (such as social support and relationships with clients) (2 studies). Five of the seven studies reported that case management in community aged care interventions significantly improved psychological health or well-being in the intervention group, while all the three studies consistently reported fewer unmet service needs among the intervention participants. In contrast, available studies reported mixed results regarding client physical or cognitive functioning and carer stress or burden. There was also limited evidence indicating significant effects of the interventions on the other client and carer outcomes as described above.
\end{abstract}

Conclusions: Available evidence showed that case management in community aged care can improve client psychological health or well-being and unmet service needs. Future studies should investigate what specific components of case management are crucial in improving clients and their carers' outcomes.

Keywords: Case management, Community aged care, Effects, Systematic review

\footnotetext{
* Correspondence: c.you@student.unimelb.edu.au; d.dunt@unimelb.edu.au

${ }^{1}$ Centre for Health Policy, Programs and Economics (CHPPE), Melbourne School of Population Health, The University of Melbourne, Melbourne, Victoria 3010, Australia

Full list of author information is available at the end of the article
} 


\section{Background}

The Case Management Society of Australia defines case management as "a collaborative process of assessment, planning, facilitation and advocacy for options and services to meet an individual's holistic needs through communication and available resources to promote quality cost-effective outcomes [1]. Case management is also described as a type of care delivery that has a long history of being applied in various settings, such as aged care, disability care, mental health and health care [2]. It first emerged in nursing practice in the 1800s and then was applied in social work practice in 1863 in the U.S. $[3,4]$.

New concepts of case management addressing complex, fragmented, duplicative and uncoordinated systems arose in the 1960s in the U.S. These originated in both the mental health movement and professional social work [5]. Further associated developments in the 1970s, again in the U.S. included deinstitutionalization, the independent living movement, increased number of community-dwelling older people with complex care needs, the fragmented care delivery system, and the need for cost control [6-8]. Many developed countries, such as England, Canada and Australia, are now attempting to integrate case management approaches into their aged care systems to provide comprehensive services for community-based frail elderly people.

There is no standard definition of case management in community aged care [9]. Compared with case management in other community-based care settings (such as primary care and community mental health), distinguishing features of case management in community aged care include [10-12]:

- Providing a broad span of case-managed community care and medical services for those having chronic, ongoing and complex medical conditions, and agerelated disabilities, including dementia $[13,14]$

- Providing services long-term or in intense short periods before placement in residential aged care

- Involving a collaborative process with the family carer

- Employing a planned approach to achieve client outcomes with cost-efficiency

- Being based in the community aged care sector

With such a long history of service provision, it is not surprising that case management has been subject to considerable scrutiny over time through systematic reviews of its effectiveness. We have examined systematic reviews looking at case management that is applied in various community-based care settings and/or targets population with specific chronic diseases [15-24]. These reviews investigated a wide range of outcome domains related to care clients, carers, care organizations (e.g. service use and costs), and care delivery systems (such as care accessibility and continuity). Nevertheless, no systematic reviews to date have specifically evaluated the effects of case management in community aged care on client and carer outcomes.

As described above, case management in community aged care differs from the other types of case management in a number of ways. Evaluating the effects of case management interventions on various outcome domains concurrently may result in heterogeneity of research findings. At this stage, a more specific, targeted review of effects is warranted. Client and carer outcomes are important effect indicators, but have been attracting less research focus compared with the other outcome measures, such as service use and costs. Therefore, we conducted this systematic review with the aim summarizing the evidence for the effects of case management in community aged care on client and carer outcomes.

\section{Methods}

Randomized control trials (RCTs) and comparative observational studies were included in this study. Due to the heterogeneity in study design, participants, interventions, outcome measures and measurement tools among studies, we conformed to the PRISMA Statement in conducting our systematic review (rather than a metaanalysis) [25]. We summarized the effects of case management in community aged care interventions based on whether the majority of studies reported significant, positive outcomes that favored the intervention group. Where the majority of available studies (in particular those with higher quality) reported that the intervention group had statistically favorable outcomes (such as greater satisfaction and better functioning status) compared with those of the control group, we reported in the results below that case management in community aged care interventions had significant effects on these outcome measures.

Study selection criteria included: no restriction on date; English language; only involving community-dwelling frail older people (suffering from age-related health problems, such as functional disabilities and dementia) and/or carers; case management interventions (excluding disease management programs that target older adults with specific chronic diseases, and specific preventive measures, such as in-home visit); care setting limited to community aged care (excluding the other communitybased care settings, such as primary care, community mental health, etc.); case management as an independent intervention (rather than as a small component of a multi-faceted intervention or an integrated care delivery system/model); published in refereed journals or publications of equivalent standard; RCTs or comparative 
observational studies; and evaluating client and/or carer outcomes.

Based on previous studies [26-30], we focused on the following outcome variables:

- Client outcomes included mortality/survival days, physical or cognitive functioning, medical conditions, psychiatric symptoms and associated behavioral problems, unmet service needs, psychological health or well-being (related to self-perceived health status, such as depression, stress, anxiety, life satisfaction etc.), and satisfaction with care.

- Carer outcomes included stress or burden, psychological health or well-being, satisfaction with care, and social consequences (such as social support, and relationships with care clients-getting on well or not).

\section{Search strategy}

We searched Web of Science, Scopus, Medline, CINAHL (EBSCO) and PsycINFO (CSA) from inception to July 2011. We also used Google Scholar to identify studies that did not appear in these databases. Table 1 shows the Medical subject heading $(\mathrm{MeSH})$ search terms and keywords. The Medline search strategy was applied to the other databases where Title/keywords/abstract was available. Reference lists of the retrieved articles and those systematic reviews as mentioned above were also checked to find potential articles.

\section{Data extraction and synthesis}

We downloaded all searched studies into EndNote 4.0 software. The first author (EY) independently screened titles and abstracts of all originally searched articles (3704 in total). If doubt existed, the second author (DD) reviewed the abstracts. Following this step, EY and DD reviewed abstracts and/or full texts of all potential, relevant articles (141 in total). EY reviewed full texts of most articles at least once. DD reviewed abstracts and where necessary full texts of these articles. After this process, EY and DD compared their results.

EY and DD independently extracted information on the characteristics (country of origin, sample size, participants, length of follow-up and intervention details), and client and carer outcomes of the studies.

Divergence regarding data extraction and synthesis was addressed through discussion between EY and DD.

\section{Quality assessment}

EY and DD also independently assessed the quality of included studies by using a checklist (see Table 2) that was informed by previous systematic reviews, the PRISMA
Table 1 Summary of Medline Search strategy

\begin{tabular}{|c|c|}
\hline 1 & (case management), key term \\
\hline 2 & exp case management/or exp managed care program/ \\
\hline 3 & (care management),key term \\
\hline 4 & $\begin{array}{l}\text { exp nursing care/or exp managed care program/or } \\
\text { exp self care }\end{array}$ \\
\hline 5 & 1 or 2 or 3 or 4 \\
\hline 6 & $\begin{array}{l}\text { ((care coordination) or (channeled care) or (care advocacy) } \\
\text { or (care integration) or (integrated care) or (key worker) } \\
\text { or (service broker) or (community matron) or linkage } \\
\text { or brokerage), key term }\end{array}$ \\
\hline 7 & 5 or 6 \\
\hline 8 & (community care), key term \\
\hline 9 & exp community networks/ \\
\hline 10 & (respite care), key term \\
\hline 11 & exp respite care/ \\
\hline 12 & (home care), key term \\
\hline 13 & $\begin{array}{l}\text { exp foster home care/or exp home care services/or } \\
\text { exp home nursing/ or home care agencies/ or home } \\
\text { health aides/or exp patient-centered care/or exp delivery } \\
\text { of health care, integrated }\end{array}$ \\
\hline 14 & (long-term care ), key term \\
\hline 15 & exp long-term care/or exp insurance, long-term care \\
\hline 16 & (home health), key term \\
\hline 17 & exp home care services/or home health aides/ \\
\hline 18 & $\left(\right.$ social service $\left.^{*}\right)$, key term \\
\hline 19 & exp social welfare/ or social work/ \\
\hline 20 & $\begin{array}{l}8 \text { or } 9 \text { or } 10 \text { or } 11 \text { or } 12 \text { or } 13 \text { or } 14 \text { or } 15 \text { or } 16 \\
\text { or } 17 \text { or } 18 \text { or } 19\end{array}$ \\
\hline 21 & $\begin{array}{l}\text { (community aged care) or (day care)or(home assistance) } \\
\text { or (home help) or(in-home) or (community-based care) } \\
\text { or (home-based care) or (aged care) or (senior care) } \\
\text { or (elder* care) or (social care), key term }\end{array}$ \\
\hline 22 & 20 or 21 \\
\hline 23 & 7 and 22 \\
\hline 24 & $\begin{array}{l}\text { limit } 23 \text { to ((English language; people aged } 65 \text { or over, } \\
\text { or } 80 \text { or over; and human) }\end{array}$ \\
\hline 25 & 23 and 24 \\
\hline
\end{tabular}

Statement and the Cochrane Handbook for Systematic Reviews of Interventions [23-25,31].

Written informed consent was obtained from the patient for publication of this report and any accompanying images.

\section{Results}

The study selection process yielded 3704 articles in total (see Figure 1). After the exclusion process, 141 full-text articles were retrieved and reviewed, with 15 studies finally included in this review. The 15 selected studies involving 13 case management in community aged care programs were summarized in Table 3 [30,32-45]. Ten studies were from the USA and one each from England, 
Table 2 Quality assessment of the studies

\begin{tabular}{|c|c|c|c|c|c|c|c|c|}
\hline \multirow[t]{2}{*}{ Author } & \multirow[t]{2}{*}{ Overall quality } & \multicolumn{7}{|c|}{ Methodology quality } \\
\hline & & $\begin{array}{l}\text { Quality } \\
\text { control }\end{array}$ & Randomization & Comparability & $\begin{array}{l}\text { Follow-up } \\
\text { rates }\end{array}$ & Dropout & $\begin{array}{l}\text { Blinding } \\
\text { assessor }\end{array}$ & Analysis \\
\hline Lam (2010) & High quality & + & + & + & $90.2 \%$ & + & + & + \\
\hline Yordi (1997) & Moderate quality & + & + & + & $34.0 \%$ & + & $?$ & $?$ \\
\hline Newcomer (1999) & Low quality & + & + & + & $36.0 \%$ & $?$ & $?$ & $?$ \\
\hline Applebaum (1988) & Low quality & $?$ & $?$ & $?$ & $?$ & $?$ & $?$ & $?$ \\
\hline Rabiner (1995) & Low quality & - & $?$ & $?$ & $100 \%$ & + & $?$ & $?$ \\
\hline Lowenstein (2000) & Moderate quality & + & + & + & $95.0 \%$ & + & $?$ & $?$ \\
\hline Eloniemi-Sulkava (2001) & Moderate quality & + & + & + & $52.0 \%$ & + & $?$ & $?$ \\
\hline Applebaum (2002) & Moderate quality & + & + & + & $82.9 \%$ & + & $?$ & $?$ \\
\hline Shapiro (2002) & Moderate quality & + & + & + & $50.0 \%$ & + & - & $?$ \\
\hline Challis (1985) & Low quality & + & - & + & $47.0 \%$ & + & $?$ & $?$ \\
\hline Marek (2006) & Low quality & + & - & + & $71.8 \%$ & + & - & $?$ \\
\hline Specht (2009) & Low quality & + & - & - & $34.9 \%$ & + & - & $?$ \\
\hline Onder (2007) & Low quality & + & - & - & $71.6 \%$ & + & - & $?$ \\
\hline Miller (1985) & Low quality & - & - & - & $?$ & $?$ & $?$ & $?$ \\
\hline Marshall, (1999) & Low quality & + & $?$ & - & $91.5 \%$ & + & - & $?$ \\
\hline
\end{tabular}

\section{Note:}

Quality control: Whether case management interventions were described clearly;

Group comparability: Whether baseline characteristics of the intervention and control groups were similar;

Follow -up rate: Whether the percentage of follow-up was complete;

Dropouts: Whether dropouts were clearly enumerated and/or compared with those completed cases at baseline;

Blinding assessor: Whether assessment was conducted by independent interviewers blinded to group or objective outcomes;

Analysis: Whether intention-to-treat analysis was applied

"+" means "yes", "_" means "no", "?" means "no details"

High quality studies: providing full information on all the seven items (follow-up rate being over $90 \%$ was regarded as "full information"); moderate quality

studies: providing information on at least four items; low quality studies: providing information on fewer than four items.

Hongkong, Finland, Italy and Israel. Only four studies were published after 2005[30,41-43].

As shown in Table 3, there were ten RCTs and five comparative observational studies in this area. Studies varied in their designs, the nature of case management interventions, their specific outcome variables and measurement tools. In these 15 studies, follow-up periods ranged from one to three years, and sample size varied from 60 to 8095 .

All the studies were based on demonstration/pilot programs that targeted community-residing elderly people with some age-related health problems (such as functional disabilities and dementia) and/or their carers.

As reported by these studies, case management in community aged care interventions generally included assessment, care planning, care plan implementation, care coordination, monitoring, and reassessment. Where programs targeted people with dementia and their family carers, specific intervention components could include education and counseling services, carer training, medical treatment and medication management, crisis interventions, client empowerment, and client advocacy. The two programs in the USA-the Channeling Demonstration and Evaluation program [34,35] and the Alzheimer's
Disease Demonstration program[32,33], increased financial benefits and allowed case managers to make independent decisions on resource allocation. Comparators were usual care for the control group and case management interventions for the experimental group.

According to Table 2, there was one high-quality RCT study (providing information on the seven items-followup rate reaching over $90 \%$ was regarded as "full information") [30], five moderate-quality studies (providing information on at least four items), and nine low-quality studies (providing information on fewer than four items).

\section{Intervention effects on the client}

14 studies reported client outcomes, including mortality/ survival days (7 studies), physical or cognitive functioning (6 studies), medical conditions (2 studies), psychiatric symptoms and associated behavioral problems (2 studies), unmet service needs (3 studies), psychological health or well-being (7 studies), and satisfaction with care (4 studies).

While mortality, physical or cognitive functioning, medical conditions, psychiatric symptoms and associated behavioral problems, and unmet service needs are 


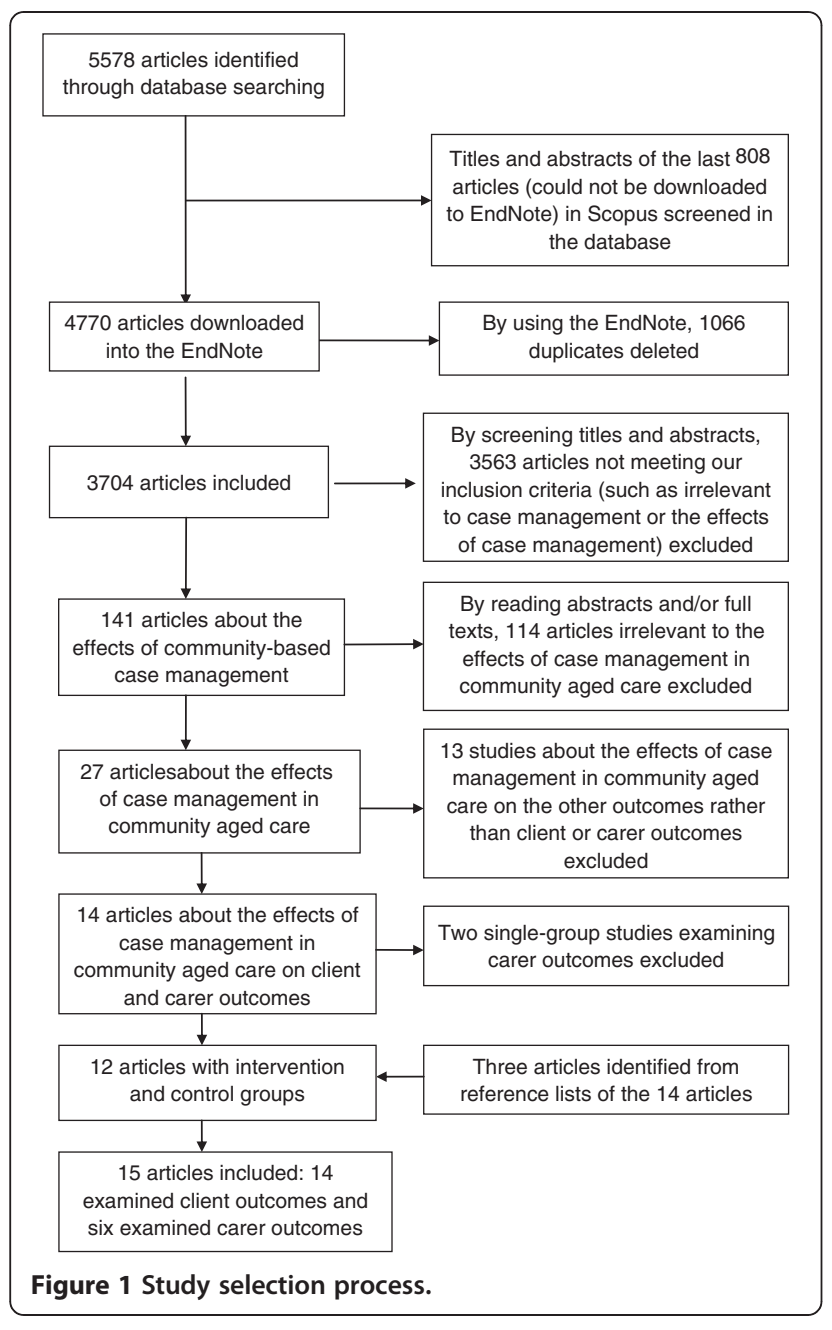

objective measures, psychological health or well-being and satisfaction with care are more subjective indictors.

\section{Mortality/survival days}

Of the seven studies examining mortality/survival days, two reported a significant effect of case management in community aged care interventions (hereafter case management interventions) on reducing client mortality or lengthening survival days.

One moderate-quality RCT reported that participants in the intervention group were significantly less likely to die or be admitted to residential care during the 18-month study period [39], while another low-quality quasi-experimental study found that the intervention group increased longevity significantly during the first and second years [44]. The other five studies all (including one low-quality RCT, one moderate-quality RCT, two low-quality quasiexperimental studies and one low-quality retrospective cohort study) reported no significant intervention-control group differences in client mortality of their different study periods $[34,38,40,41,43]$.

\section{Physical or cognitive functioning}

Six studies investigated clients' physical functional status and/or cognitive functioning, but reported inconsistent results across different measures during different study periods. For example: Some studies found that case management interventions could significantly improve the performance of ADL-related tasks (but not IADLrelated tasks or cognitive status measured by GDS/ MMSE) among the intervention participants; others showed that the interventions only had significant effects in the long-term rather than in the short-term.

Three low-quality studies provided some evidence that case management interventions had a long-term effect on ADL measures. One low-quality RCT found that participants in the intervention group had significantly fewer number of ADL disabilities during the six months, 7-12 months, and 12-18 months of follow-up respectively [34]. This study, however, found no significant intervention-control group differences in the number of IADL disabilities or the number of days restricted to bed during these study periods [34]. Another low-quality RCT found no significant intervention-control group difference in client functional status after one year, but reported significantly better functional status among intervention participants after two years [45]. A third low-quality quasi-experimental study reported that participants in the intervention group had significantly better ADL performance after 12 months, but indicated no significant intervention-control group differences in physical or cognitive status after six months [41].

Three other studies, however, indicated no clear effect of case management interventions on ADL or cognitive functioning. One moderate-quality RCT reported no significant intervention-control group differences in the number of ADL or IADL disabilities during sixmonth and 12-month follow-up periods respectively [38]. One high-quality RCT reported no significant intervention-control group differences in client cognitive status during any study periods [30]. And one lowquality quasi-experimental study showed no significant intervention-control group differences in ADL/IADL index, minimum-mental state examination (MMSE), or GDS during the 3-9 months and 9-15 months of followup [42].

\section{Medical conditions}

Of the two studies examining client medical conditions, one low-quality quasi-experimental study reported less pain and dyspnoea among participants in the intervention group during the 12-month follow-up period [41], while the other moderate-quality RCT showed no significant intervention-control group differences in client health status during six-month, 12-month and 18-month study periods [38]. 
Table 3 Characteristics of studies included in the systematic review

\begin{tabular}{|c|c|c|c|c|c|}
\hline $\begin{array}{l}\text { Author, year \& } \\
\text { location }\end{array}$ & $\begin{array}{l}\text { Study design, study sample } \\
\text { (mean age of older pepole X } \\
\text { where applicable), sample size } \\
\text { (N) \& intervention length }\end{array}$ & $\begin{array}{l}\text { Case management } \\
\text { in community aged care } \\
\text { interventions }\end{array}$ & Measurement instrument & Client outcomes & Carer outcomes \\
\hline $\begin{array}{l}\text { Yordi (1997) } \\
\text { USA }\end{array}$ & $\begin{array}{l}\text { RCT Older people with } \\
\text { dementia Intervention/control: } \\
X=78.3 / X=78.3 ; N=2,707 / \\
N=2,5473 \text { years }\end{array}$ & $\begin{array}{l}\text { Needs assessment, assisting } \\
\text { carers arranging services, } \\
\text { activating care plan \& care } \\
\text { quality monitoring. Intervention: } \\
\text { smaller caseload ( } \mathrm{n}=30) \& \text { higher } \\
\text { monthly benefits }(\$ 430-\$ 699 \text { per } \\
\text { client), control: larger caseload } \\
(\mathrm{n}=100) \& \text { lower monthly } \\
\text { benefits ( } \$ 290-\$ 489 \text { per client) } \\
\text { (the Alzheimer's disease } \\
\text { demonstration program). }\end{array}$ & $\begin{array}{l}\text { Functional status: measured } \\
\text { by a version of the Katz ADL \& } \\
\text { Lawton and Brody's IADL scale at six- } \\
\text { month intervals }\end{array}$ & $\begin{array}{l}\text { Robust effect on reducing unmet } \\
\text { needs with ADL/IADL tasks over time } \\
\text { Significantly fewer unmet service } \\
\text { needs in the intervention group } \\
\text { during different follow-up periods }\end{array}$ & \\
\hline $\begin{array}{l}\text { Newcomer } \\
\text { (1999) USA }\end{array}$ & $\begin{array}{l}\text { RCT Carers of older people with } \\
\text { dementia Intervention/control: } \\
X=63 / X=63 ; N=2,731 / N=2,576 \\
3 \text { years }\end{array}$ & See [32]. & $\begin{array}{l}\text { Burden: measured by an adapted } \\
\text { scale developed by Zarit, Reever and } \\
\text { Bach-Peterson Depression: measured } \\
\text { by the short-form Geriatric } \\
\text { Depression Scale }\end{array}$ & & $\begin{array}{l}\text { Burden: no significant } \\
\text { intervention-control } \\
\text { group differences during } \\
6 \text {-, 12- or } \\
\text { 36-month follow-up } \\
\text { Depression: no significant } \\
\text { intervention-control } \\
\text { group differences during } \\
\text { 12- or 36-month } \\
\text { follow-up }\end{array}$ \\
\hline $\begin{array}{l}\text { Applebaum } \\
\text { (1988) USA }\end{array}$ & $\begin{array}{l}\text { RCT Older people (eligible for } \\
\text { nursing home admission) \& } \\
\text { carers Intervention/control: } \\
N=1,861 / N=2,01318 \text { months }\end{array}$ & $\begin{array}{l}\text { Needs assessment, care planning, } \\
\text { service arrangement, monitoring, } \\
\text { care plan modification \& } \\
\text { reassessment. Intervention: case } \\
\text { managers having control over } \\
\text { pooled funds; control: adopting a } \\
\text { brokerage model (the } \\
\text { Channeling Demonstration } \\
\text { program). See [46] }\end{array}$ & $\begin{array}{l}\text { Mortality rates Functioning: } \\
\text { measured by an ADL five- item } \\
\text { (eating, transfer, toileting, dressing, } \\
\text { bathing \& continence) scale, an IADL } \\
\text { seven-item (housekeeping, meal } \\
\text { preparation, shopping, } \\
\text { transportation, taking medicine, } \\
\text { financial management \& telephone } \\
\text { use) scale, and number of days } \\
\text { restricted to bed Client social/ } \\
\text { psychological well-being: overall life } \\
\text { satisfaction, morale, attitude towards } \\
\text { to aging, social interactions, self- } \\
\text { perceived health\& overall } \\
\text { contentment index Carer social/ } \\
\text { psychological well-being: life } \\
\text { satisfaction \& relationship with } \\
\text { clients Carer stressors: perceived } \\
\text { emotional/physical/financial strain \& } \\
\text { number of stressful behavior } \\
\text { problems }\end{array}$ & $\begin{array}{l}\text { No significant intervention-control } \\
\text { group differences in mortality rates } \\
\text { during different follow-up periods } \\
\text { (first six months, 7-12 months \& 12- } \\
18 \text { months) Significantly higher life } \\
\text { satisfaction, fewer number of unmet } \\
\text { service needs \& fewer number of } \\
\text { ADL disabilities in the intervention } \\
\text { group during different follow-up } \\
\text { periods No significant intervention- } \\
\text { control group differences in the } \\
\text { number of IADL disabilities \& } \\
\text { number of days restricted to bed } \\
\text { during different follow-up periods }\end{array}$ & $\begin{array}{l}\text { Satisfaction with service } \\
\text { arrangements improved } \\
\text { significantly over time No } \\
\text { significant intervention- } \\
\text { control group differences } \\
\text { in satisfaction, social well- } \\
\text { being/psychological \& } \\
\text { stressor measures during } \\
\text { different follow-up } \\
\text { periods }\end{array}$ \\
\hline $\begin{array}{l}\text { Rabiner (1995) } \\
\text { USA }\end{array}$ & $\begin{array}{l}\text { RCT People aged } 65 \text { and over } \\
\text { Six-month analysis: } \mathrm{N}=2,237 \text {; } \\
\text { 12-month analysis: } \mathrm{N}=1,726\end{array}$ & See $[46]$ & $\begin{array}{l}\text { Satisfaction: measured by extent of } \\
\text { confidence (not confident, } \\
\text { somewhat confident and very }\end{array}$ & $\begin{array}{l}\text { No significant intervention-control } \\
\text { group difference in satisfaction } \\
\text { during six-month follow-up } \\
\text { Significantly greater satisfaction in }\end{array}$ & \\
\hline
\end{tabular}

Significantly greater satisfaction in 
Table 3 Characteristics of studies included in the systematic review (Continued)

\begin{tabular}{|c|c|c|c|c|c|}
\hline & $\begin{array}{l}\text { (no details about the sample } \\
\text { size of each group) } 1 \text { year }\end{array}$ & & $\begin{array}{l}\text { confident) in care at six- \& } \\
12 \text {-month follow-up }\end{array}$ & $\begin{array}{l}\text { the intervention group during } 12- \\
\text { month follow-up }\end{array}$ & \\
\hline $\begin{array}{l}\text { Lowenstein } \\
\text { (2000) Israel }\end{array}$ & $\begin{array}{l}\text { RCT People aged } 69 \text { and older } \\
\text { (most of whom suffering from } \\
\text { physical, cognitive and mental } \\
\text { diseases) \& carers Intervention/ } \\
\text { control: } \mathrm{N}=30 / \mathrm{N}=301 \text { year }\end{array}$ & $\begin{array}{l}\text { Referral, intake, needs } \\
\text { assessment, care plan activation, } \\
\text { care linkage, care plan } \\
\text { modification, informal care } \\
\text { support, monitoring, } \\
\text { reassessment, evaluation \& } \\
\text { discharge arrangement. }\end{array}$ & $\begin{array}{l}\text { Outcomes: reported by social } \\
\text { workers through individual/group } \\
\text { interviews at the end of the study }\end{array}$ & $\begin{array}{l}\text { Significant improvements in } \\
\text { community participation, satisfaction } \\
\text { with services, consumer choice \& } \\
\text { unmet } \\
\text { needs in the intervention } \\
\text { group. }\end{array}$ & $\begin{array}{l}\text { Significant improvements } \\
\text { in satisfaction with } \\
\text { services \& burden in the } \\
\text { intervention group. }\end{array}$ \\
\hline $\begin{array}{l}\text { Eloniemi-Sulkava } \\
\text { (2001) Finland }\end{array}$ & $\begin{array}{l}\text { RCT People aged } 65 \text { and older } \\
\text { with dementia Intervention/ } \\
\text { control: } X=78.8 / X=80.1 ; \\
N=53 / N=472 \text { years }\end{array}$ & $\begin{array}{l}\text { Advocacy, counseling, annual } \\
\text { training, follow-up calls, in-home } \\
\text { visits, care arrangement \& 24- } \\
\text { hour services. }\end{array}$ & Year $1 \& 2$ death rates & $\begin{array}{l}\text { No significant intervention-control } \\
\text { group differences in death rates } \\
\text { during different follow-up periods }\end{array}$ & \\
\hline $\begin{array}{l}\text { Applebaum } \\
\text { (2002) USA }\end{array}$ & $\begin{array}{l}\text { RCT Older people with } \\
\text { disabilities Intervention/control: } \\
X=78.2 / X=79.5 ; N=154 / N=154 \\
18 \text { months }\end{array}$ & $\begin{array}{l}\text { Preventive activities (assessment } \\
\text { \& consumer training) \& } \\
\text { intervention activities } \\
\text { (communication with physicians } \\
\text { \& hospital discharge planning). }\end{array}$ & $\begin{array}{l}\text { Death rates \& mean number of } \\
\text { survival days by six, } 12 \text { and } 18 \\
\text { months Functioning status: } \\
\text { measured by average number of } \\
\text { ADL disabilities (bathing, dressing, } \\
\text { transfer from bed to chair, getting to } \\
\text { the toilet, eating\& inside mobility), } \\
\text { average number of IADL disabilities } \\
\& \text { cognitive disorder (having } \\
\text { Alzheimer/Dementia/ other cognitive } \\
\text { or not) at different time points } \\
\text { (baseline, six- \& } 12 \text {-month follow-up) } \\
\text { Overall health status (range } 0-16 \text { ), } \\
\text { overall health and service satisfaction } \\
\text { (range 0-20): measured at different } \\
\text { time points }\end{array}$ & $\begin{array}{l}\text { No significant intervention-control } \\
\text { group differences in death rates, } \\
\text { mean number of survival days, } \\
\text { service satisfaction, health status \& } \\
\text { physical functioning during different } \\
\text { follow-up periods }\end{array}$ & \\
\hline $\begin{array}{l}\text { Shapiro (2002) } \\
\text { USA }\end{array}$ & $\begin{array}{l}\text { RCT Older adults on waiting list to } \\
\text { receive social care Intervention/ } \\
\text { control: } X=77.8 / X=76.9 ; N=40 / \\
N=6518 \text { months }\end{array}$ & $\begin{array}{l}\text { Geriatric assessment subsequent } \\
\text { service provision, care planning, } \\
\text { care coordination \& contacts per } \\
3 \text { months. }\end{array}$ & $\begin{array}{l}\text { Nursing home admission/death rate } \\
\text { by } 18 \text { months Quality of life } \\
\text { (including depression, social } \\
\text { satisfaction, mastery \& life } \\
\text { satisfaction): measured at three- } \\
\text { month intervals }\end{array}$ & $\begin{array}{l}\text { The intervention group was } \\
\text { significantly less likely to die or be } \\
\text { institutionalized Significantly better } \\
\text { quality of life in the intervention } \\
\text { group during different follow-up } \\
\text { periods }\end{array}$ & \\
\hline $\begin{array}{l}\text { Miller (1985) } \\
\text { USA }\end{array}$ & $\begin{array}{l}\text { Quasi-experimental study Low- } \\
\text { income elderly medical people at } \\
\text { risk of institutionalization } \\
\text { Intervention/control: } X=79 / X=76.5 \\
\& N=1068 / \mathrm{N}=1495 \text { in community } \\
\text { group. } X=77 / X=76.1 \& N=983 / \\
N=848 \text { in hospital group. } X=80.9 / \\
X=81.8 \& N=261 / N=196 \text { in nursing } \\
\text { home group. } X=77.9 / X=90 \& \\
N=607 / N=28 \text { in target group } 2 \\
\text { years }\end{array}$ & $\begin{array}{l}\text { A team of case managers } \\
\text { providing multi-dimensional } \\
\text { assessment, care planning, } \\
\text { service arrangement, follow-up } \\
\text { and reassessment }\end{array}$ & $\begin{array}{l}\text { Number of days of life saved within } \\
\text { one year and two years respectively }\end{array}$ & $\begin{array}{l}\text { Significantly increased longevity } \\
\text { among participants in the } \\
\text { intervention group by } 3.97 \text { days in } \\
1981, \text { and } 7.25 \text { days } 1982 \\
\text { Interventions were most effective for } \\
\text { the frailest clients }\end{array}$ & \\
\hline
\end{tabular}

RCT Older adults on waiting list to receive social care Intervention/ control: $X=77.8 / X=76.9 ; \mathrm{N}=40$

Quasi-experimental study Low income elderly medical people at risk of institutionalization discharge arrangement.

Preventive activities (assessment (communication with physicians cognitive disorder (having or not) at different time points (baseline, six- \& 12-month follow-up) nth intervals multi-dimension ment, care planning,

arangement, follow-up participants in the 1981, and 725 days 1982 Interventions were most effective for the frailest clients 
Table 3 Characteristics of studies included in the systematic review (Continued)

Marshall, (1999) RCT People aged 65 and over

USA $\mathrm{N}=159 / \mathrm{N}=1602$ years

RCT People aged 65 years old or above with mild dementia Intervention/control: $X=78.6 /$ $X=78.2 ; \mathrm{N}=59 / \mathrm{N}=4318$ months One year (with 4 months' intervention)
Challis (1985) England
Quasi-experiment Older people eligible for nursing home admission \& carers Intervention control: $\mathrm{N}=74 / \mathrm{N}=743$ years
Screening, selection, assessment treatment plan, service arrangement within and outside the program \& periodic reassessment

Assessment and advice, homebased program on cognitive stimulation, liaising with other care professionals to ensure clients and carers' participation community activities

\section{Functional status: measured by ADLs (bathing, eating, transferring, \\ toileting \&dressing rated from 1 to 3 . higher score meant higher dependency level) and IADLs (needing telephoning, transportation walking, \& medication services or not) at baseline, year $1 \&$ year 2 Perceived health status: measured on a scale of 1 to 4 defined as excellent good, fair, or poor respectively at baseline, year $1 \&$ year 2 Satisfaction with care: measured on a scale of 1 to 5 from very satisfied to very dissatisfied at baseline, year 1 \& year 2}

Carer stress: measured by Zarit Carer Burden Interview (ZBI) (22 items, specifically including perceived health, psychological well-being, financial impact, social life, and care and client relationships) at threemonth intervals (below is the same) Carer psychological health: measured by general health questionnaire (GHQ) Carer subjective quality of life: measured by personal well-being Index for Adults (PWI-As) Client cognitive status: measured by Min Mental State Examination (CMMSE) Client psychiatric symptoms and behavioral disturbance: measured by The Neuropsychiatric Inventory (NPI) Client personal well-being: measured by the Personal Well-Being Indexby the Personal Well-Being Index-
Intellectual Disability (PWI-ID) Client depression: measured by Cornell Scale for Depression in Dementia (CSDD)

Referrals, assessment, care planning, monitoring \& case closure.
Death rates: measured at one-year intervals Client quality of life (morale depressed mood, anxiety, loneliness \& felt capacity to cope) \& Carer outcomes (level of subjective burden extent of strain menta health difficulties; difficulties in socia life, household routine,

employment\& financial issues): measured at the end of the study

During one-year follow-up:

significantly improved perceived health status in the intervention group; control group was more satisfied with care; no significan intervention-control group differences in functional status measures During two-year study period: significantly better functiona status in the intervention group Time effects: the intervention group did not experience significant changes in functional status or perceived health status during the whole study period compared with the control group

Significant improvement in client depression in the intervention group during 4-month follow up No group differences in carer differences in client cognitive status, health, or subjective client psychiatric symptoms and quality of life during any behavioral disturbances, or persona well-being during any study periods study periods

No significant intervention-control group difference in death rate by 24 months; significantly lower death rate in the intervention group by 36 months. Significantly better quality of life (except anxiety) in the intervention group
Significantly lower level of burden in the

intervention group No significant interventioncontrol group differences in the other carer outcomes 
Table 3 Characteristics of studies included in the systematic review (Continued)

\begin{tabular}{|c|c|c|c|c|c|}
\hline $\begin{array}{l}\text { Marek (2006b) } \\
\text { USA }\end{array}$ & $\begin{array}{l}\text { Quasi-experiment The frail elderly } \\
\text { aged } 64 \text { and older Intervention/ } \\
\text { control: } \mathrm{N}=55 / \mathrm{N}=301 \text { year }\end{array}$ & $\begin{array}{l}\text { Needs assessment, care plan } \\
\text { reviewing, monitoring \& hospital } \\
\text { care coordination }\end{array}$ & $\begin{array}{l}\text { Functioning status: measured by } \\
\text { ADL (bed mobility, transfers, } \\
\text { locomotion, eating \& toileting. Each } \\
\text { item scored from 0-4) score, } \\
\text { cognitive performance scale (range } \\
0-6 \text { ) at different time points } \\
\text { (baseline, six- \& 12-month follow-up) } \\
\text { Depression rating scale (range 0-2 } \\
\text { for observed frequency of each of } \\
\text { the seven mood indicators): } \\
\text { measured at different time points } \\
\text { Frequency of health outcomes } \\
\text { measured at different time points: } \\
\text { including incontinence (range 0-2), } \\
\text { pain (range 0-3), dyspnea (range 0- } \\
\text { 4) \& ability of medication } \\
\text { management (range } 0-1 \text { ) }\end{array}$ & $\begin{array}{l}\text { More death cases ( } 6 \text { vs.2) by } 12 \\
\text { months in the intervention group. } \\
\text { No significant intervention-control } \\
\text { group differences in all client } \\
\text { outcomes during six months } \\
\text { Significantly better ADL performance, } \\
\text { and less pain \& dysnea in the } \\
\text { intervention group during } 12 \\
\text { months. }\end{array}$ & \\
\hline $\begin{array}{l}\text { Specht (2009) } \\
\text { USA }\end{array}$ & $\begin{array}{l}\text { Quasi-experiment Older people } \\
\text { with dementia \& carer } \\
\text { Intervention/control: } X=82.4 / \\
X=78.5 ; \mathrm{N}=167 / \mathrm{N}=821.5 \text { months }\end{array}$ & $\begin{array}{l}\text { Needs identification and } \\
\text { assessment, care plan } \\
\text { development, home visits, } \\
\text { monthly phone contacts, } \\
\text { quarterly face-to-face contacts, } \\
\text { periodic reassessment \& care } \\
\text { system coordination. }\end{array}$ & $\begin{array}{l}\text { Outcomes of 3-9 months \& 9-15 } \\
\text { months were assessed Client } \\
\text { functioning: measured by MMSE } \\
\text { (range 1-30), GDS (range 1-7), } \\
\text { functional Assessment II, Groff, R.L } \\
\text { (range 1-3), modified IADL/ADL's } \\
\text { from Lawton and Brody (range 1-5) } \\
\text { Client behaviors: measured by a } \\
\text { rating checklist (Garrity and Klein) } \\
\text { Client \& carer health status: } \\
\text { measured by SF-36 Carer well-being, } \\
\text { stressors \& endurance potential: } \\
\text { measured by NOC (Moorehead et al.) }\end{array}$ & $\begin{array}{l}\text { Significant decline in ADL abilities in } \\
\text { the intervention group from baseline } \\
\text { to each follow-up No significant } \\
\text { intervention-control group } \\
\text { differences in ADL disabilities, MMSE, } \\
\text { GDS \& behavior rating index during } \\
\text { different follow-up periods. }\end{array}$ & $\begin{array}{l}\text { Significantly lower stress, } \\
\text { and better endurance } \\
\text { potential \& well-being in } \\
\text { the intervention group } \\
\text { during different follow-up } \\
\text { periods }\end{array}$ \\
\hline $\begin{array}{l}\text { Onder (2007) } \\
\text { Italy }\end{array}$ & $\begin{array}{l}\text { Retrospective cohort Frail elderly } \\
\text { people Intervention/control: } \\
\mathrm{X}=82.1 / \mathrm{X}=82.5 ; \mathrm{N}=1,184 / \mathrm{N}= \\
2,1081 \text { year }\end{array}$ & $\begin{array}{l}\text { Initial assessment, monitoring, } \\
\text { additional care provision, care } \\
\text { plan design and implementation, } \\
\text { care arrangement \& care } \\
\text { coordination. }\end{array}$ & 1-year mortality & $\begin{array}{l}\text { No significant intervention-control } \\
\text { group difference in 1-year mortality }\end{array}$ & \\
\hline
\end{tabular}

ment \& care

measured by SF-36 Carer well-being,

inance potentia: 
Psychiatric symptoms and associated behavioral problems

Two studies (including one high-quality RCT and one low-quality quasi-experimental study) examining these outcomes did not find significant intervention-control group differences during their different study periods $[30,42]$.

\section{Unmet service needs}

Improving unmet service needs as an outcome showed more evidence for successful application of case management in community aged care. Three studies, including two moderate-quality RCTs and one low-quality RCT, consistently reported that case management interventions had significant effects on improving clients' unmet service needs $[32,34,36]$.

\section{Psychological health or well-being}

Psychological health and wellbeing had more evidence for a good outcome of case management interventions for older clients. Of the seven studies evaluating this outcome, five (including two low-quality RCTs, two moderatequality RCTs and one low-quality quasi-experimental study) reported that case management interventions had significant effects on improving intervention participants' psychological health or well-being across different measures, such as self-perceived life satisfaction, morale, depression, mastery, and personal health status $[34,36,39,40,45]$.

One high-quality RCT reported no significant intervention-control group differences in clients' personal well-being, but showed significant improvement in depression among participants in the intervention group during different study periods [30].

The remaining one low-quality quasi-experimental study revealed no significant intervention-control group difference in client depression after six months [41].

\section{Satisfaction with care}

Four studies examined this outcome, with three reporting no significant effects of case management interventions on improving client satisfaction with care services.

One moderate-quality study found that the intervention group improved satisfaction with service provision during one-year study period [36]. One low-quality RCT documented significantly higher satisfaction among participants in the intervention group during the 12-month study period, but found no significant interventioncontrol group difference during the six months of follow-up [35].

In contrast, one moderate-quality RCT demonstrated no significant intervention-control group differences at any study periods [38], while another low-quality RCT found that the control group had significantly higher satisfaction with care during one-year study period [45].

\section{Intervention effects on the carer}

Six studies reported carer outcomes, including carers' stress or burden (6 studies), satisfaction with care (2 studies), psychological health or well-being (including perceived health conditions, life satisfaction, psychological distress, depression etc.) (5 studies), and social consequences (such as social support and relationships with clients) (2 studies). All these outcome measures, as we observe, are related to carers' subjective feelings.

\section{Stress or burden}

The six studies examining this outcome reported variable effects of case management interventions on carer stress or burden.

One moderate-quality RCT reported significant improvement in the burden of carers in the intervention group during the one-year study period [36]. Another two low-quality quasi-experimental studies showed significantly lower level of stress or burden among carers in the intervention group during their different study periods $[40,42]$. In contrast, two low-quality RCTs $[33,34]$ and one high-quality RCT [30] reported no significant intervention-control group differences in carer burden or stress during their different study periods.

\section{Satisfaction with care}

Of the two studies reporting carer satisfaction, one moderate-quality RCT demonstrated significantly higher satisfaction among carers in the intervention group over the one-year study period [36], while the other lowquality RCT found no significant intervention-control group differences during any study periods [34].

\section{Psychological health or well-being}

Of the five studies investigating this outcome, only one low-quality quasi-experimental study reported that participants in the intervention group had significantly better well-being during different follow-up periods [42]. Conversely, four studies (including one high-quality RCT, two low-quality RCTs, and one low-quality quasi-experimental study) did not find significant intervention-control group differences during their different study periods $[30,33,34,40]$.

\section{Social consequences}

Two studies (including one low-quality RCT and one low-quality quasi-experimental study) reported no significant intervention-control group differences in carer social consequences, such as carers' social life and carers' relationships with their clients $[34,40]$.

\section{Discussion}

Community care is increasingly the preferred mode of care for older people to avoid residential care. While 
consumer-directed care for this section of the population is gaining popularity, a large proportion of older people will continue to use case managers to assist them in negotiating their care needs [15]. This review provided largely consistent evidence that case management interventions improve older clients' psychological health or well-being and also deliver significant improvements in unmet service needs. Clear effects of the interventions on other client outcomes and carer outcomes are not so evident, with mixed evidence for the other outcome variables reviewed here. We found that studies reported inconsistent results regarding client physical or cognitive functioning and carer stress or burden. There was also limited evidence supporting that case management in community aged care interventions improve client length of survival, health conditions, behavioral problems or satisfaction with care, as well as carer satisfaction with care, psychological health or well-being and social consequences.

There are a number of limitations to these conclusions. First, the number of studies involved was small. While we identified ten RCTs, only one RCT was rated high quality according to our quality criteria [30]. One RCT did not describe the study design [34], requiring us to obtain this information elsewhere [46]. Information about blinding assessors and intention-to-treat analysis was commonly missing in most studies. Other methodological limitations of many studies include small sample size, and lack of information on sample size calculation or strategies of controlling confounding factors.

Assessment, care planning, care plan implementation, client advocacy, monitoring, review, and case closure were reported as the core case management functions in community aged care setting, but many studies did not provide full information about the intensity, breadth and duration of each function. This poses a challenge to attribute different client and carer outcomes to specific intervention components.

As with most systematic reviews, we found that variations in the nature, content and individual components of case management interventions or functions, as well as absence of information on the intervention implementation make it challenging to compare the results among different studies $[8,47]$.

The choice of outcome measures that are appropriate or valid is critical to a fair evaluation of the effects of case management in community aged interventions. The studies reviewed here used a large number of outcome measures with little justification of their appropriateness or robustness [48]. Some studies also reported that the instruments used for outcome assessment were inconsistent over time or among different research participants, again leading to challenges in drawing evidence-based conclusions [33,38].
Another prominent issue is that the studies reviewed here used diverse instruments or methods to measure different client and carer outcomes. For instance, clients' physical or cognitive functioning was measured by the number of ADL/IADL limitations, ADL/IADL score, MMSE, and/or GDS; unmet service needs were reported by clients, carers or care professionals.

An overarching question for further research is the 'dose-response' relationship between case management interventions and client and carer outcomes. Some researchers claim that more focused but less intensive or comprehensive case management in community aged care interventions can be effective $[49,50]$. Other researchers accentuate that the intensity of the interventions should be strong enough-at least different from that of the usual care-to achieve desired outcomes [32,33].

Participants in some case management in community aged care programs were not chosen with specific inclusion criteria in mind; for example, individuals with low-risk of nursing home admission were unexpectedly enrolled by some programs targeted at reducing nursing home admission. This may partly explain why the case management interventions did not achieve some desired outcomes [34]. This finding lends support to previous research, indicating that problems in recruiting suitable participants hamper many programs in demonstrating their success [5,51-53].

Although we did not find systematic reviews specifically assessing the effects of case management in community aged care interventions on client and carer outcomes, our findings, to some degree, were consistent with previous related systematic reviews that examined the effects of case management interventions on various outcomes.

First, previous reviews reported that the effects of case management interventions on many client outcomes were inconclusive. For example, one review revealed that most included studies found no significant interventioncontrol group differences in client satisfaction, physical functioning, mortality, or quality of life [54]. Regardless of different care settings, study populations, and interventions previous reviews and our study focused on, case management interventions cannot improve all client outcomes. Our findings here suggest that case management interventions alone might not reverse or significantly improve some health conditions in the frail elderly.

Second, previous reviews concluded that case management interventions have moderate or no significant effects on carer burden and depression $[24,28,29,55,56]$. One reason for this finding is that it might be difficult to improve carer outcomes in reality, since caregiving always leads to carers experiencing high levels of stress, burden and other negative consequences; or the finding could be attributed to measurement difficulties. Furthermore, 
many case management interventions include no or only moderate intervention components for carers themselves. This should be addressed in designing new case management programs in future, if carer outcomes are one of the target goals.

In general, this study answers the review question: "What are the effects of case management in community aged care interventions on carer and client outcomes?" The evidence from this review may enlighten policy makers to design appropriate case management interventions and reasonable intervention goals in the area of community aged care in future. Moreover, it may advise care professionals to focus on the areas where the interventions have significant effects, so as to make appropriate decisions on resource allocation in their practice.

\section{Limitations}

This systematic review is limited by the methodological shortcomings in most studies, e.g. nine studies were rated as low-quality studies, while five were classified as moderate-quality studies. Other limitations are as follows:

First, we did not review studies that compared different types of case management models or focus on case management as a component of multifaceted interventions. But valuable information can be obtained from multifaceted interventions, only if case management components can be separated from the whole intervention [57].

Second, we did not search studies published in nonEnglish Journals or grey literature. We have noted that most included studies were from the United States.

Finally, because of limited resources, we did not use a pre-specified protocol to guide the conduct of our systematic review. Since review of the effects of case management/ case management in community aged care on various outcomes is ongoing [58], this issue should be addressed in the future.

\section{Conclusions}

Available evidence in this review showed that case management in community aged care interventions can improve client psychological health or well-being and unmet service needs. In contrast, the effects of the interventions on client mortality, functional status, medical conditions, behavioral problems and satisfaction with care services, as well as carer outcomes as noted by this review are less conclusive.

Future studies should investigate what specific components of case management are crucial in achieving improved outcomes for the client and their carer. In addition, undertaking evaluation studies by employing rigorous study designs are warranted.

\section{Abbreviations}

(RCTs): Randomized control trials; (ADL): Activities of daily living; (IADL): Instrumental activities of daily living; (MMSE): Minimum-mental state examination; (GDS): Global dementia scale.

\section{Competing interests}

There are no competing interests.

\section{Authors' contributions}

DD\&EY had equal contributions to conceptualizing this paper, as well as screening literature, reviewing studies for inclusion, extracting data, and writing and critically reviewing the paper. CD conceptualized and critically reviewed the paper. All the authors interpreted data, read and approved the final manuscript.

\section{Authors' information}

$\mathrm{EY}$ is a PhD candidate in the Centre for Health Policy, Programs and Economics (CHPPE), School of Population Health, The University of Melbourne. Professor DD is the founding director of the Centre for Health Policy, Programs and Economics (CHPPE), School of Population Health, The University of Melbourne. He is a public health specialist and medicallytrained epidemiologist, with expertise in health program evaluation and health service research. Dr. CD is a professor of Aged Care at Australian Catholic University/Catholic Homes and the director of Service Development \& Evaluation Division in the National Ageing Research Institute (NARI). She has expertise in aged care research and health program evaluation. Dr. $\mathrm{AH}$ is a senior lecturer \& health economist in the Centre for Health Policy, Programs and Economics (CHPPE), School of Population Health, The University of Melbourne.

\section{Acknowledgement}

Many thanks to Eva Di Quinzio and Tania Celeste, the Librarians of the University of Melbourne, for assisting us with the search strategy of this review, as well as to Ms Allison Yates and Mr Chris Chiu for providing some advice to this review.

\section{Author details}

${ }^{1}$ Centre for Health Policy, Programs and Economics (CHPPE), Melbourne School of Population Health, The University of Melbourne, Melbourne, Victoria 3010, Australia. ${ }^{2}$ National Ageing Research Institute, Royal Melbourne Hospital, PO Box 2127, Melbourne, Victoria 3050, Australia. ${ }^{3}$ Australian Catholic University, 115 Victoria Pde Fitzroy, Melbourne, Victoria 3065, Australia.

Received: 18 June 2012 Accepted: 31 October 2012

Published: 14 November 2012

\section{References}

1. Case Management Society of Australia (CMSA): Case Management and Community Care: A Discussion Paper. Sydney: Case Management Society of Australia; 2006.

2. Cooper B, Deborah YR: National case management standards in Australia-purpose, process and potential impact. Aust Health Rev 2006, 30(1):12-16.

3. Gursansky DJ, Harvey J, Kennedy R: Case Management: Policy, Practice and Professional Business. Crows Nest NSW: Allen\&Unwin: Columbia University Press; 2003.

4. Tahan HA: Case management: a heritage more than a century old. Nurs Case Manag 1998, 3(2):55-60.

5. Camilleri P: Researching case management: making it a fact. The Journal of Australian Case Management 2000, 2:18-23.

6. Scharlach AE, Giunta N, Mills-Dick K: Case Management in Long-term Care Integration: An Overview of Current Programs and Evaluations. California: 2001.

7. Moxley D: The Practice of Case Management. California: Sage, Newbury Park; 1989.

8. Reilly S, Hughes J, Challis D: Case management for long-term conditions: implementation and processes. Ageing \& Society 2010, 30:125-155.

9. Schmuttermaier JR, Schmitt DJ, King CM, Gwynne AE: Whole of client health care in a gridlocked system: an insider dialogue between the theory and practice of community case management. Home Health Care Management and Practice 2011, 23(1):36-49. 
10. Taylor TJ: Case management in long-term care integration programs: an analysis of the case management in Arizona, California, Minnesota, Texas \& Wisconsin. California: PhD thesis, Touro University International, Faculty of the College Health; 2004.

11. Gruman CA: An analysis of case management programs and practices: correlates of organizational strength. Boston: PhD thesis. University of Massachusetts Boston; 1997.

12. Good Practice Guide for HACC Funded Case Management Projects. Sydney: NSW Department of Ageing, Disability and Home Care; 2006.

13. Ho PS: Effects of utilization of case management related services on the quality of llfe of the community-based long-term care population. Virginia: PhD thesis, Virginia Commonwealth University, Department of Health Administration, School of Allied Health Professions; 1993.

14. Applebaum R, Austin CD: Long-Term Care Case Management: Design and Evaluation. New York: Springer Publishing Company; 1990.

15. Low LF, Yap M, Brodaty $\mathrm{H}$ : A systematic review of different models of home and community care services for older persons. BMC Health Serv Res 2011, 11:1-15.

16. Oeseburg B, Wynia K, Middel B, Reijneveld S: Effects of case management for frail older people or those with chronic illness: a systematic review. Nurs Res 2009, 58(3):201-210.

17. Smith L, Newton R: Systematic review of case management. Aust N Z J Psychiatry 2007, 41(1):2-9.

18. Norris S, Nichols P, Caspersen C, Glasgow R, Engelgau M, Leonard J, Isham G, Snyder S, Carande KV, Garfield S, Briss P, McCullonch D: The effectiveness of disease and case management for people with diabetes. A systematic review. Am J Prev Med 2002, 22(14):15-38.

19. Grabowski D: The cost-effectiveness of noninstitutional long-term care services: review and synthesis of the most recent evidence. Medical care research and review 2006, 63(1):3-28.

20. Lupari M, Coates V, Adamson G, Crealey GE: 'We're just not getting it right'- how should we provide care to the older person with multimorbid chronic conditions? J Clin Nurs 2011, 20(9-10):1225-1235

21. Hallberg IR, Kristerisson J: Preventive home care of frail older people: a review of recent case management studies. J Clin Nurs 2004, 13(6B):112-120.

22. Oeseburg B, Jansen D, De Keyser J: Reducing discrepancies between MS patients' needs and use of healthcare services by applying a transmural care model. The Journal of neuroscience nursing: journal of the American Association of Neuroscience Nurses 2004, 36(4):214-230.

23. Pimouguet C, Lavaud T, Dartigues JF, Helmer C: Dementia case management effectiveness on health care costs and resource utilization: a systematic review of randomized controlled trials. J Nutr Health Aging 2010, 14(8):669-676

24. Eklund K, Wilhelmson K: Outcomes of coordinated and integrated interventions targeting frail elderly people: a systematic review of randomised controlled trials. Health Soc Care Community 2009, 17(5):447-458

25. Liberati A, Altman D, Telzlaff J, Mulrow C, Gtzsche P, Loannidis J, Clarke M, Devereaux PJ, Kleijnen J, Moher D: The PRISMA statement for reporting systematic reviews and meta-analyses of studies that evaluate health care interventions: explanation and elaboration. J Clin Epidemio/ 2009, 62(10):e1-e34.

26. Gaugler JE, Yu F, Krichbaum K: Predictors of nursing home admission for persons with dementia. Medical Care 2009, 47(2):191-197.

27. Robinson L, Lliffe S, Brayne C, Goodman C, Rait G, Manthorpe J: Primary care and dementia: 2. long-term care at home: psychosocial interventions, information provision, carer support and case management. Int J Geriatr Psychiatry 2009, 25(7)):657-664.

28. Brodaty $H$, Green A, Koschera A: Meta-analysis of psychosocial interventions for caregivers of people with dementia. J Am Geriatr Soc 2003, 51(5):657-664

29. Chien LY, Chu H, Guo JL, Liao YM, Chang LI, Chen CH, Chou KR: Caregiver support groups in patients with dementia: a meta-analysis. Int J Geriatr Psychiatry 2011, 26(10):1089-1098.

30. Lam L, Lee J, Chung J, Lau A, Woo J, Kwok T: A randomized controlled trial to examine the effectiveness of case management model for community dwelling older persons with mild dementia in Hong Kong. Int J Geriatr Psychiatry 2010, 25(4):395-402.

31. In Cochrane Handbook for Systematic Reviews of Interventions 4.2.6. Edited by Higgins JPT, Green S. Chichester, UK: Wiley; 2006.
32. Yordi C, DuNah R, Bostrom A, Fox P, Wilkinson A, Newcomer R: Caregiver Supports: Outcomes from the Medicare Alzheimer's Disease Demonstration. Health Care Financ Rev 1997, 19(2):97-116.

33. Newcomer R, Yordi C, DuNah R, Fox P, Wilkinson A: Effects of the Medicare Alzheimer's Disease Demonstration on Caregiver Burden and Depression. Health Serv Res 1999, 34:669-689.

34. Applebaum RA, Christianson JB, Harrigan M, Schore J: The evaluation of the National Long Term Care Demonstration:The effect of channeling on mortality, functioning, and well-being. Health Serv Res 1988, 23(1):143-159.

35. Rabiner DJ, Mutran E, Stearns SC: The effect of channeling on home care utilization and satisfaction with care. Gerontologist 1995, 35(2):186-195.

36. Lowenstein A: A case management demonstration project for the frail elderly in Israel. Care Manag J 2000, 2(1):5-14.

37. Eloniemi S, Notkola I, Hentinen M, Kivela S, Sivenius J, Sulkava R: Effects of supporting community-living demented patients and their caregivers: a randomized trial. J Am Geriatr Soc 2001, 49(10):1282-1287.

38. Applebaum R, Straker J, Mehdizadeh S, Warshwa G, Gothelf E: Using highintensity care management to integrate acute and long-term care services: substitute for large scale system reform?. Care Manag J 2002, 3(3):113-119.

39. Shapiro A, Taylor M: Effects of a community-based early intervention program on the subjective well-being, institutionalization, and mortality of low-income elders. Gerontologist 2002, 42(3):334-341.

40. Challis $D$, Davies $B$ : Long term care for the elderly: the community care scheme. Br J Soc Work 1985, 15(6):563-579.

41. Marek K, Popejoy L, Petroski G, Rantz M: Nurse care coordination in community-based long-term care. J Nurs Scholarsh 2006, 38(1):80-86

42. Specht J, Bossen A, Hall GR, Zimmerman B, Russell J: The effects of a dementia nurse care manager on improving caregiver outcomes. Am J Alzheimers Dis Other Demen 2009, 24(3):193-207.

43. Onder G, Liperoti R, Soldato M, Carpenter I, Steel K, Bernabei R: Case management and risk of nursing home admission for older adults in home care: results of the Aged in home care study. J Am Geriatr Soc 2007, 55(3):439-444.

44. Miller LS, Clark ML, Clark WF: The comparative evaluation of California's multipurpose senior services project. Home Health Care Serv Q 1985, 6(3):49-79.

45. Marshall BS, Long MJ, Voss J, Demma K, Sterl KP: Case management of the elderly in a health maintenance organization: The implications for program administration under managed care. J Healthc Manag 1999, 44(6):477-493.

46. Kemper $\mathrm{P}$ : The evaluation of the national long term care demonstration: overview of the findings. Health Serv Res 1988, 23(1):161-174.

47. Agency for Healthcare Research and Quality, U.S. Department of Health and Human Services: Comparative Effectiveness of Case Management for Adults with Medical IIIness and Complext Care Needs: Comparative Effectiveness Review. Rockville: Agency for Healthcare Research and Quality (AHRQ); 2011.

48. Young VS, Fine M: Case Management in Community Aged Care: Mapping the Landscape in NSW. Sydney: Centre for Research on Social Inclusion Macquarie University; 2010

49. Cox C, Albisu K: The Alzheimer's Connections Demonstration Program: instituting a national case management program. Am J Alzheimers Dis Other Demen 2001, 16(5):279-284.

50. Beswick A, Rees K, Dieppe P, Ayis S, Gooberman HR, Horwood J: Complex interventions to improve physical function and maintain independent living in elderly people: a systematic review and meta-analysis. Lancet 2008, 371(9614):725-735

51. Summers M: Great expectations: a policy case study of four case management programs in one organization. Melbourne, Victoria: PhD thesis, The University of Melbourne, Faculty of Arts; 2007.

52. Howe AC, Doyle C, Wells Y: Targeting in community care for frail older people: a review of recent literature and analysis of the aged care assessment program minimus data set. Melbourne: Australian Institute for Primary Care, Faculty of Health Sciences, La Trobe University; 2006.

53. The Department Advisory Committee: Better Targeting for HACC, Linkages and Community Aged Care Packages in Victoria-Some Considerations: A discussion paper from the Victorian departmental advisory committee on the home and community care program. Victoria: Department of Health; 2003. 
54. Hallberg I, Kristensson J: Preventive home care of frail older people: a review of recent case management studies. J Clin Nurs 2004,

13(6B):112-120.

55. Smits CHM, De-Lang J, Droes RM, Meiland F, Vernooij-Dassen M, Pot AM: Effects of combined intervention programmes for people with dementia living at home and their caregivers: a systematic review. Int J Geriatr Psychiatry 2007, 22(12):1181-1193.

56. Schoenmakers B, Buntinx F, DeLepeleire J: Supporting the dementia family caregiver: The effect of home care intervention on general well-being. Aging Ment Health 2010, 14(1):44-56.

57. Zwarenstein M, Reeves S, Straus SE, Pinfold P, Goldman J: Case management: effects on professional practice and health care outcomes (protocol). The Cochrane Collaboration 2009, 3(1):1-6.

58. Smith S, Soubhi H, Fortin M, Hudon C, O'Dowd T: Interventions for improving outcomes in patients with multimorbidity in primary care and community settings. Cochrane Database Syst Rev 2012, 4:CD006560CD006560.

doi:10.1186/1472-6963-12-395

Cite this article as: You et al:: Effects of case management in community aged care on client and carer outcomes: a systematic review of randomized trials and comparative observational studies. BMC Health Services Research 2012 12:395.

\section{Submit your next manuscript to BioMed Central and take full advantage of:}

- Convenient online submission

- Thorough peer review

- No space constraints or color figure charges

- Immediate publication on acceptance

- Inclusion in PubMed, CAS, Scopus and Google Scholar

- Research which is freely available for redistribution 